\title{
The rhomboid protease family: a decade of progress on function and mechanism
}

Sinisa Urban* and Seth W Dickey

\begin{abstract}
Summary
Rhomboid proteases are the largest family of enzymes that hydrolyze peptide bonds within the cell membrane. Although discovered to be serine proteases only a decade ago, rhomboid proteases are already considered to be the best understood intramembrane proteases. The presence of rhomboid proteins in all domains of life emphasizes their importance but makes their evolutionary history difficult to chart with confidence. Phylogenetics nevertheless offers three guiding principles for interpreting rhomboid function. The near ubiquity of rhomboid proteases across evolution suggests broad, organizational roles that are not directly essential for cell survival. Functions have been deciphered in only about a dozen organisms and fall into four general categories: initiating cell signaling in animals, facilitating bacterial quorum sensing, regulating mitochondrial homeostasis, and dismantling adhesion complexes of parasitic protozoa. Although in no organism has the full complement of rhomboid function yet been elucidated, links to devastating human disease are emerging rapidly, including to Parkinson's disease, type II diabetes, cancer, and bacterial and malaria infection. Rhomboid proteases are unlike most proteolytic enzymes, because they are membrane-immersed; understanding how the membrane immersion affects their function remains a key challenge.
\end{abstract}

\section{Gene organization and evolutionary history}

Proteolysis within the membrane was discovered in seemingly rare contexts nearly 15 years ago [1-3]. It is now widely appreciated that this fascinating regulatory paradigm permeates most areas of modern cell biology [4-7]. Of the three protease families that catalyze

*Correspondence: surban@jhmi.edu

Howard Hughes Medical Institute, Department of Molecular Biology and Genetics, Johns Hopkins University School of Medicine, Baltimore, MD 21205, USA intramembrane proteolysis, rhomboid enzymes are the only family that were not discovered from the direct study of human disease. The name 'rhomboid' has its origin deep in the rich folklore of Drosophila genetics. Rhomboid emerged from the historic quest to identify all genes required to organize construction of a free-living organism from a single cell $[8,9]$. Because genes were named after the altered appearance of the mutant larval cuticle, the mis-shaped, rhombus-like head skeleton of the mutant embryo earned rhomboid its name. Mutating the growth factor that rhomboid activates yielded indistinguishable head-skeleton defects, and was named spitz ('pointed' in German).

The rhomboid gene was cloned and sequenced by Bier and colleagues in 1990, revealing a seven transmembrane (7TM) protein with no homology to any sequence known at the time [10]. The spitz sequence was more informative, encoding a clear epidermal growth factor (EGF)like protein [11]. The fact that rhomboid mirrored spitz phenotypically [9], and encoded a seven TM protein, led to the proposal that it might be a serpentine receptor for Spitz signaling [11]. But as sequencing of genomes from diverse organisms began to reveal rhomboid homologs in every form of cellular life [12], it became clear that rhomboid proteins may be at the core of very diverse biological regulation.

Sequence analysis, however, yielded no clues about the underlying biochemical function of rhomboid proteins, and no other homologs were as well studied as Drosophila rhomboid. A decade of Drosophila genetics had, however, set the stage for a biochemical approach: rhomboid was definitively implicated as an upstream activator of Spitz in the signal-sending cell, providing a framework for analyzing its molecular function [12-15]. A focused analysis of Spitz activation eventually yielded four key pieces of the puzzle [16]: (i) substochiometric levels of Rhomboid triggered Spitz proteolysis, implying that Rhomboid acts enzymatically; (ii) proteolysis depends absolutely only on four Rhomboid residues, and their identity is consistent with serine protease catalysis; (iii) Spitz proteolysis is blocked only by serine protease inhibitors; and (iv) Spitz is cleaved within its TM segment at a depth similar to that of the putative rhomboid 
catalytic serine. These pieces fit together into a model in which rhomboid acts as an intramembrane serine protease for Spitz [16], which was confirmed 4 years later by reconstituting cleavage with pure proteins [17-19].

The ability to study Spitz proteolysis as a direct test of rhomboid activity was used to determine that even distant bacterial homologs are functional intramembrane serine proteases [20]. Most bacterial species are now known to encode one rhomboid protease, while some encode two, and very few encode three [21]. Rhomboid proteases are also present in many if not most Archaea, but the greatest expansion occurred in multicellular organisms and some parasitic protozoa. Although the human, mouse and Drosophila genomes encode at least seven rhomboid genes, the largest number of rhomboid genes are encoded by plants (13 in Arabidopsis), which do not have EGF signaling [21-23]. In many of these diverse organisms, at least one rhomboid has directly been demonstrated to have proteolytic activity (Table 1) [16,20,23-26].

Rhomboid proteases are found in all branches of life, yet the sequence identity across all family members is strikingly low, around only $6 \%[12,21,22]$. We suggest that this is not despite rhomboid proteases being so widespread but because of it. This divergence is exacerbated by their sequences being predominantly transmembrane and thus experiencing a different evolutionary pressure [27]. This has made phylogenetic analyses noisy, yielding few incontrovertible conclusions and inevitably fueling debate. Of particular intrigue is their evolutionary origin: rhomboid proteins have been argued to be perhaps the most widely distributed membrane proteins in nature [21] (Figure 1). This near ubiquity is instinctively viewed as evidence of an ancient enzyme family that evolved early [12]. Although this is likely if the last universal common ancestor already encoded several different rhomboid proteins, phylogenetic analysis has also raised the possibility of a different history in which rhomboid proteins are a later invention of bacteria that rapidly spread to most other organisms [21]. This scenario requires a controversial amount of horizontal gene transfer to populate all kingdoms of life. Currently the true nature of rhomboid phylogenetic history remains a point of inconsolable debate, but three observations serve as valuable guiding principles.

\section{Characteristic structural features}

The first organizing principle emerging from sequence analysis is the observation that rhomboid proteases come in three distinct topological flavors (Figure 2) [21]. The simplest consists of the 6TM core, which itself is the smallest catalytically active unit. This form predominates in bacteria, but is also represented, albeit more rarely, in eukaryotic organisms, including animals. To this basic unit eukaryotes add a seventh TM segment following the $6 \mathrm{TM}$ core $(6+1 \mathrm{TM}$ form). Five of the seven Drosophila, human, and mouse rhomboid proteins are of this form. Analogous 7TM forms also occur in bacteria, but are rare. Lastly, a distinct form of 7TM rhomboid proteases exists in endosymbiotic organelles, adding the seventh $\mathrm{TM}$ preceding the $6 \mathrm{TM}$ core $(1+6 \mathrm{TM}$ form $)$. The best studied are those imported into mitochondria [28-30], although interest in plastid-resident rhomboid proteins has recently been sparked [31]. Although the sequence analysis is clear on these three topological distinctions, their functional relevance is unclear. The expectation is that they confer different biochemical properties, although current evidence, albeit limited, indicates that many bacterial 6TM forms and eukaryotic $6+1 \mathrm{TM}$ forms show similar activity against surrogate substrates, including Spitz [17,32,33].

Although protease activity has been reconstituted with both the 6TM and 6+1TM rhomboid forms in vitro, it is only a 6TM form called GlpG from Escherichia coli and Haemophilus influenzae that has proven amenable to structural analysis [34-37]. This major breakthrough - the first atomic-resolution structure of any intramembrane protease - not only confirmed that proteolysis is intramembrane and catalyzed by a serine protease apparatus, but revealed an unanticipated and complex architecture. Although a thorough description is beyond the scope of the current discussion (see [38] for a comprehensive review), two features are characteristic (Figure 3): although most TM helices are long and run roughly perpendicular to the membrane, the fourth TM segment runs slanted relative to the others and enters the center of the protein as an extended loop, converting to an $\alpha$ helix at the catalytic serine. More unexpected was the orientation of the long L1 loop connecting TMs 1 and 2, which forms a lateral hairpin that lies half submerged in the membrane. This feature, which has not been encountered before or since, has major structural implications and results in a highly asymmetric protein. It is assumed that the structure of the other rhomboid forms will be analogous, and recent modeling of the mitochondrial 1+6TM form on E. coli GlpG hints at an unanticipated level of similarity [39].

In addition to the number of TMs, two further variations provide potential for additional rhomboid diversity. First, in all three forms, the cytosolic amino termini are highly variable, ranging from large domains to being nonexistent. The implications, however, remain unclear, at least partly because achieving well-diffracting crystals required absence of this domain, making its relationship to the catalytic core speculative. On the simplest level, these domains may house sorting signals [40].

Secondly, rhomboid proteins are often encountered that clearly lack catalytic residues. These should be 
Table 1. Known rhomboid protease substrates and functions across evolution

\begin{tabular}{|c|c|c|c|c|}
\hline Rhomboid & Organism & Substrate & Function & References \\
\hline Rho-1 & Drosophila & Spitz, Keren & EGFR signaling (embryo, most developing tissues, adult) & {$[8-16,89]$} \\
\hline Rho-2/Stet & Drosophila & Gurken,? & EGFR signaling in oocyte, germline & {$[89,90]$} \\
\hline Rho-3/Ru & Drosophila & Spitz, Keren & EGFR signaling in embryo, eye disc & {$[12,89,91]$} \\
\hline Rho-4 & Drosophila & Spitz, Keren? & EGFR signaling? & [89] \\
\hline Rho-7 & Drosophila & DmOpa1-like DmPINK1 & Mitochondrial fusion, mitophagy & {$[30,55]$} \\
\hline CeROM1 & C. elegans & LIN-3L & Amplifying EGFR signaling (vulva) & [47] \\
\hline AarA & P. stuartii & TatA & Activating twin-arginine translocon, quorum sensing & {$[20,17,61-62,66]$} \\
\hline GlpG & E. coli & $?$ & $?$ & $\begin{array}{c}{[17-20,34-36} \\
77-78,83-85,88]\end{array}$ \\
\hline HiGlpG & H. influenzae & $?$ & $?$ & {$[37,81]$} \\
\hline YqgP & Bacillus subtilis & $?$ & $?$ & {$[17,18,20]$} \\
\hline PA3086 & Pseudomonas aeruginosa & $?$ & $?$ & {$[17,18,20]$} \\
\hline AqRho & Aquifex aeolicus & $?$ & $?$ & {$[17,20]$} \\
\hline Pcp1 & Saccharomyces cerevisiae & Mgm1, Сcp1 & Mitochondrial membrane fusion, pre-sequence removal & {$[28,52-54]$} \\
\hline PARL & Homo sapiens Mus musculus & PINK1, others & $\begin{array}{l}\text { Downregulating mitophagy, crista remodeling, } \\
\text { anti-apoptosis }\end{array}$ & {$[28,29,56-60]$} \\
\hline AtRBL2 & Arabidopsis thaliana & $?$ & $?$ & [23] \\
\hline RHBDL2 & H. sapiens & TM, EphrinB1,2,3, EGF & Blood clotting? Cell migration? Cancer? & {$[24,48,87]$} \\
\hline PfROM4 & P. falciparum & EBA175, TRAP, other adhesins & Invasion & {$[26,69]$} \\
\hline PfROM1 & Plasmodium sp. & AMA1? & Invasion? Growth & {$[26,69,70]$} \\
\hline TgROM1 & Toxoplasma gondii & $?$ & Growth & {$[25,40,68,75]$} \\
\hline TgROM2 & T. gondii & $?$ & $?$ & {$[25,40,68]$} \\
\hline $\operatorname{TgROM} 3$ & T. gondii & $?$ & $?$ & {$[25,68]$} \\
\hline TgROM4 & Toxoplasma gondii & AMA1, MIC2, MIC6 & Invasion & {$[25,68,71,72]$} \\
\hline TgROM5 & Toxoplasma gondii & AMA1, MIC2, MIC6 & Invasion & {$[25,26]$} \\
\hline EhROM1 & Entamoeba histolytica & Lectins & Phagocytosis, immune evasion & {$[73,74]$} \\
\hline
\end{tabular}

Only those rhomboid proteases whose proteolytic activity has been detected are included in the table. A question mark'?' denotes unknown function. EGFR, epidermal growth factor receptor.

considered rhomboid proteins but not rhomboid proteases. Two predominant clusters are a distinct $6+1 \mathrm{TM}$ form in animals, called iRhom proteins [22,41], and a $6 \mathrm{TM}$ form that is represented widely in eukaryotes by the Derlin proteins [42-45]. Both of these have been implicated in endoplasmic reticulum-associated degradation (ERAD). Derlins have clear sequence homology near the membrane-submerged L1 loop, but also less conspicuously along their entire length, and are thus likely to adopt a GlpG-like 6TM structure. Although clearly not proteolytic, their potential similarity to other aspects of the rhomboid protease mechanism should not be discounted at this early stage (but lie beyond the scope of this review).

\section{Localization and function}

The second guiding principle stems from the tremendous diversity of organisms that encode rhomboid enzymes. Since these include organisms that do not encode any known forms of cell-to-cell communication, sequence information implies that rhomboid proteins perform an ancient and fundamental role in cell biology. This function is not essential for cell survival, however, because several lineages are missing rhomboid genes entirely, presumably by gene loss [21]. Although defining the cellular functions of rhomboid proteases has proven a persistent challenge, focused investigations have succeeded in documenting the function of at least one rhomboid in nearly a dozen organisms (Table 1). These functions are usually regulated by substrate trafficking, and fall into four broad categories (Figure 4).

First, rhomboid proteases initiate animal cell signaling by releasing growth factors from the membrane. This function emerged from detailed genetic study of Drosophila development; rhomboid proteases are localized in the Golgi apparatus and act as the signal-generating component by cleaving Spitz to initiate the pathway in neighboring cells $[16,46]$. Although a role in regulating 


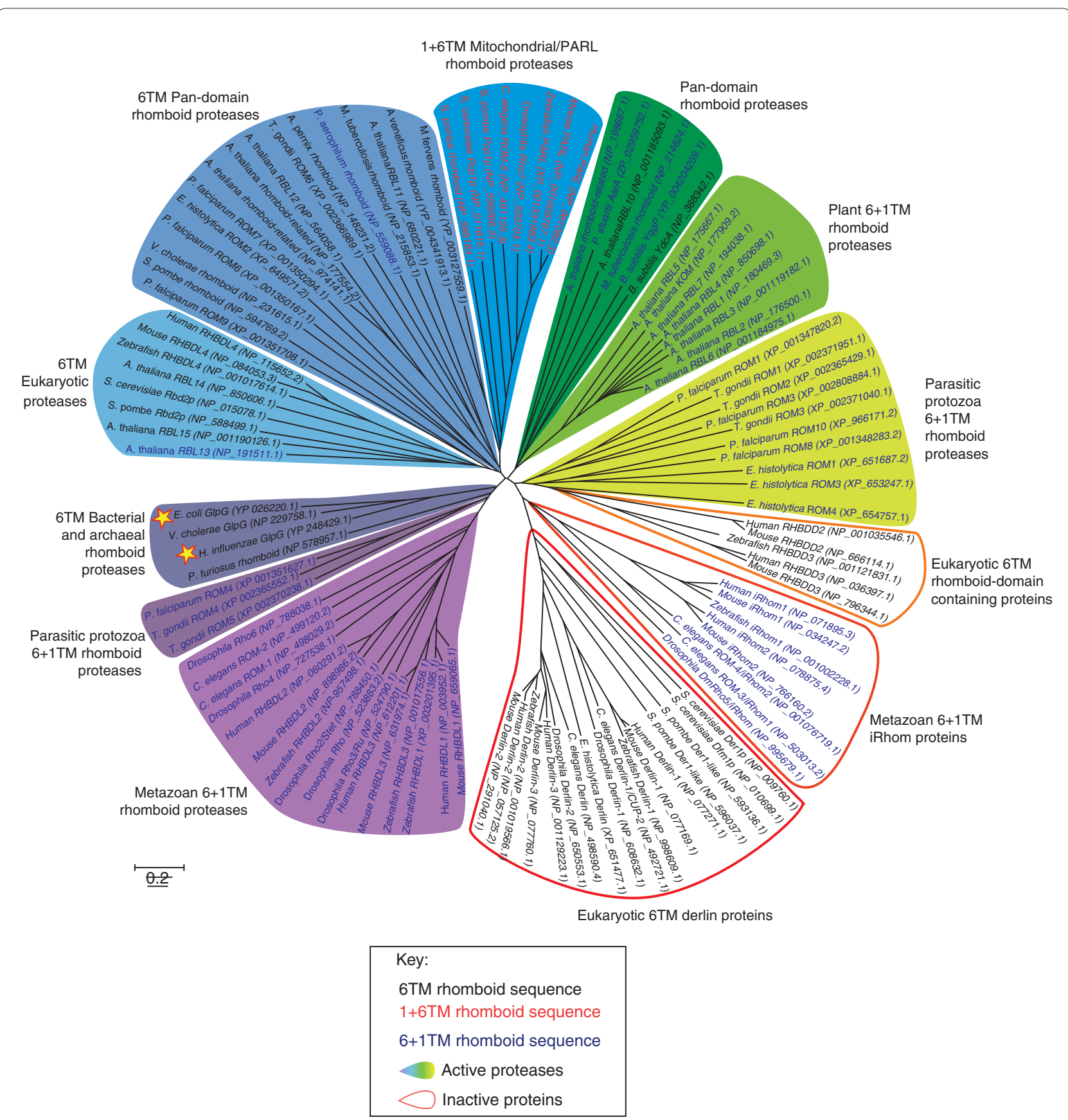

Figure 1. Phylogenetic tree of rhomboid proteins. Rhomboid protein sequences are widely scattered throughout all branches of cellular life. A subset of 109 Rhomboid and Derlin family protein reference sequences, retrieved from the NCBI RefSeq database, was chosen to illustrate their diversity. Mega 5.05 was used to align sequences by MUSCLE and construct an unrooted neighbor-joining phylogenetic tree. Branches are labeled according to their common characteristics and are shaded or outlined to denote active or inactive protease sequences, respectively. Individual sequence names are colored black, blue, or red to indicate a 6TM, 6+1TM, or 1+6TM arrangement, respectively, with each RefSeq accession number included within parentheses. Despite the tremendous number and diversity of rhomboid proteins, structures of only two 6TM rhomboid proteases have been solved (yellow stars).

EGF signaling is also seen in Caenorhabditis elegans vulval development, CeROM-1 has a surprisingly minor role as a target of EGF signaling that sets up a paracrine loop to amplify and spread the signal [47]. Even less is clear in mammals: recent investigations have localized rhomboid proteins to the secretory pathway and cell surface and begun to uncover increased rhomboid expression in cancer cells with potential links to growth 


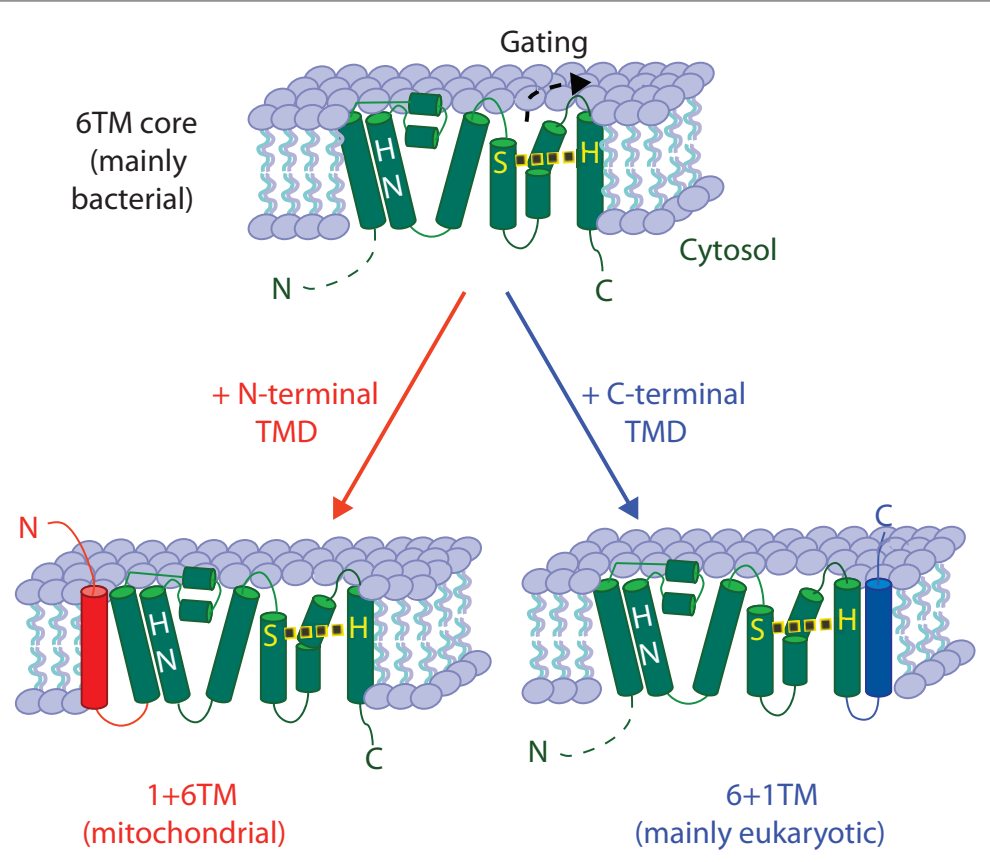

Figure 2. Rhomboid proteins exist in three topological forms. The smallest, catalytically active form of a rhomboid protease consists of a 6TM core, with variable amino termini (dashed lines). Most eukaryotic and mitochondrial rhomboid proteases have an additional TM segment, added either carboxy-terminally (eukaryotes, blue) or amino-terminally (mitochondria, red) to the 6TM core, as depicted. Catalytic residues are in yellow for nucleophilic chemistry (hydrolysis) and white for electrophilic residues (oxyanion transition state stabilization). Cytoplasm is down in each diagram.

factor signaling $[24,48,49]$. However, this is not limited to active rhomboid proteases; expression of the iRhom RHBDF1, which is localized in the endoplasmic reticulum in human epithelial cancer cells, increased secretion of the EGF ligand transforming growth factor- $\alpha$ [50]. Accordingly, RHBDF1 silencing decreased pathway activation through EGF receptor (EGFR), ERK and AKT phosphorylation, and limited tumor growth in mice [51]. The Drosophila homolog, however, was recently found to have the opposite effect of decreasing EGFR signaling by promoting the ERAD-mediated degradation of EGF ligands [41]. The basis of this remarkable discrepancy is currently unclear; knockout mouse studies are expected to provide clarity on the physiological roles of rhomboid proteins.

Recent studies have also placed the mitochondrial rhomboid protease at the nexus of key pathways that govern mitochondrial fusion, mitophagy and apoptosis. All mitochondrial rhomboid proteins are encoded in the nuclear genome, and imported into mitochondria. The main function of the mitochondrial rhomboid Pcp1 is to release the dynamin-like GTPase Mgm1 from the membrane [28,52,53]. Because Mgm1 is essential for mitochondrial fusion and Mgm1 cleavage occurs only in healthy mitochondria, this limits fusion to occurring between healthy organelles [54]. A similar function was described in Drosophila [30], but genetic interactions soon revealed further complexity in metazoans; the mitochondrial rhomboid DmRho-7 also participates in the Parkin/PINK1 pathway that malfunctions in Parkinson's disease [55]. It has recently become clear that the human mitochondrial rhomboid PARL cleaves PINK1 to suppress its ability to recruit the Parkin ubiquitin ligase onto mitochondria [56-58]. Without PARL cleavage, PINK1 accumulates in mitochondria and fails to be recruited properly to damaged mitochondria. A PARL knockout mouse suffers tremendous atrophy several months after birth resulting from malformed mitochondria and elevated apoptosis, although without mitochondrial fusion defects [29]. PARL has also been implicated in suppressing apoptosis in lymphocytes, potentially through a different substrate, High-temperature regulated A (HtrA, also called Omi) [59]. Intriguingly, mutations in PARL have recently been found in Parkinson's disease patients [58] and diabetes patients [60], although the significance of these mutations for disease remains speculative.

The third category of rhomboid function was revealed in Providencia stuartii, a Gram-negative bacterial pathogen. Genetic screens identified its rhomboid homolog, AarA, to be required for production of an unidentified signal for quorum sensing $[61,62]$. Once the similarity to rhomboid was noted [63], proteolytic activity of AarA was demonstrated against Spitz [20], and AarA was found to partially rescue tissue development of Drosophila 


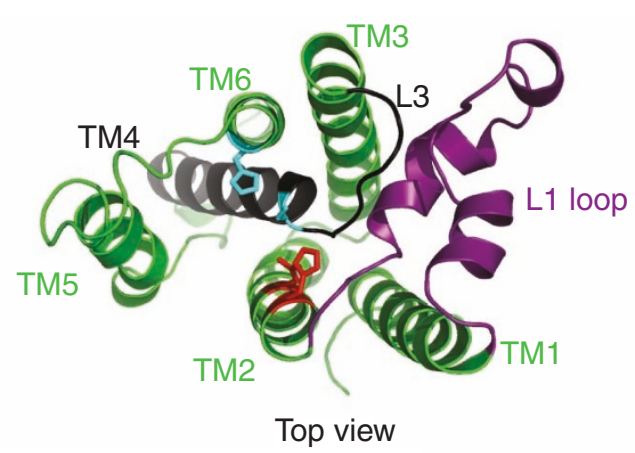

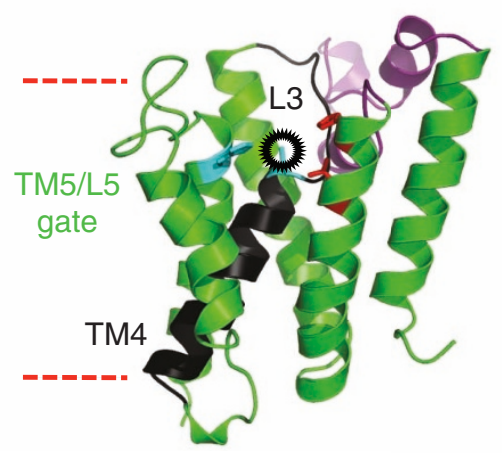

Lateral view (back)

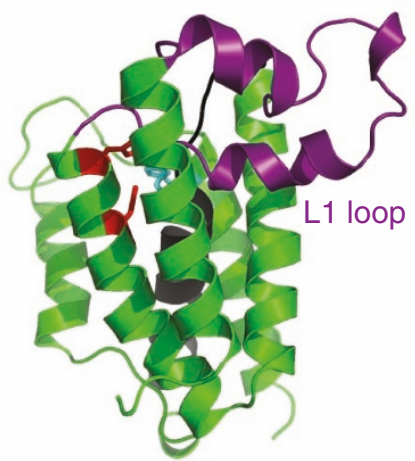

Lateral view (front)

Figure 3. Structural features of the rhomboid 6TM core. The crystal structure of the 6TM core of the E. coli rhomboid protease GlpG (PDB 2NRF molecule A) is shown from three vantage points ('top view' is looking at the cell from the outside with the membrane in the plane of the page). The protein forms a compact helical bundle, with two characteristic features. A short and slanted TM4 (black) forms a helix below the catalytic serine (circled in the 'back' view), but an extended loop (L3) above it. This slanted trajectory and extended loop create a cavity above the serine. The L1 loop (purple) forms a hairpin structure that nestles between TMs 1 and 3 and protrudes laterally into the outer leaflet of the membrane (red dashed lines representing the membrane interface are provided only for reference). Catalytic dyad residues serine and histidine are in cyan; putative oxyanion-stabilizing electrophilic asparagine and histidine residues are in red.

mutant in rhomboid [64]. Historically, the intriguing similarity of activating Drosophila EGF signaling and producing an auto-inducer for bacterial quorum sensing, both by a rhomboid, received much attention $[63,65]$. But the similarity proved to be superficial when the substrate was identified to be TatA, a component of the twinarginine translocation machinery [66]. As such, AarA removes a short amino-terminal extension, presumably to activate the machinery for signal secretion, rather than activating the signal itself. TatA from other bacteria, including E. coli, lacks this short extension and is immediately active, and the AarA function is therefore an exception. Nevertheless, this is the only known function for a rhomboid protease in any prokaryote, and it dramatically highlights the apparent diversity of rhomboid function even within similar bacteria.

Finally, rhomboid proteases help to dismantle adhesive junctions in unicellular eukaryotic parasites. This is the only role that was discovered by searching for rhomboid targets using substrate specificity determinants [33]. The adhesins of Plasmodium and Toxoplasma are necessary for host-cell invasion, making them essential proteins for the survival of these obligate intracellular parasites [67]. These parasites encode six or more rhomboid proteases, two of which in each organism are known to process these adhesins at the end of the invasion program $[25,26$, 68-70]. The precise need for this dismantling is not entirely clear, but has been thought to free the parasite from being tethered to the host plasma membrane. Recent knockdown experiments indicate that this processing is important for efficient invasion [71], although the full extent is incompletely understood and may involve later functions during parasite replication within the host cell [72]. Even the noncell-invasive Entamoeba histolytica encodes a highly active rhomboid protease, which is localized to the parasite surface but which relocalizes to phagosomes during feeding and the bud neck during immune evasion, perhaps to shed surface proteins, including lectins [73,74]. The functions of other Plasmodium or Toxoplasma rhomboid proteases not involved in invasion are not yet understood [75], and many other parasites encode rhomboid enzymes whose functions have never been explored. 


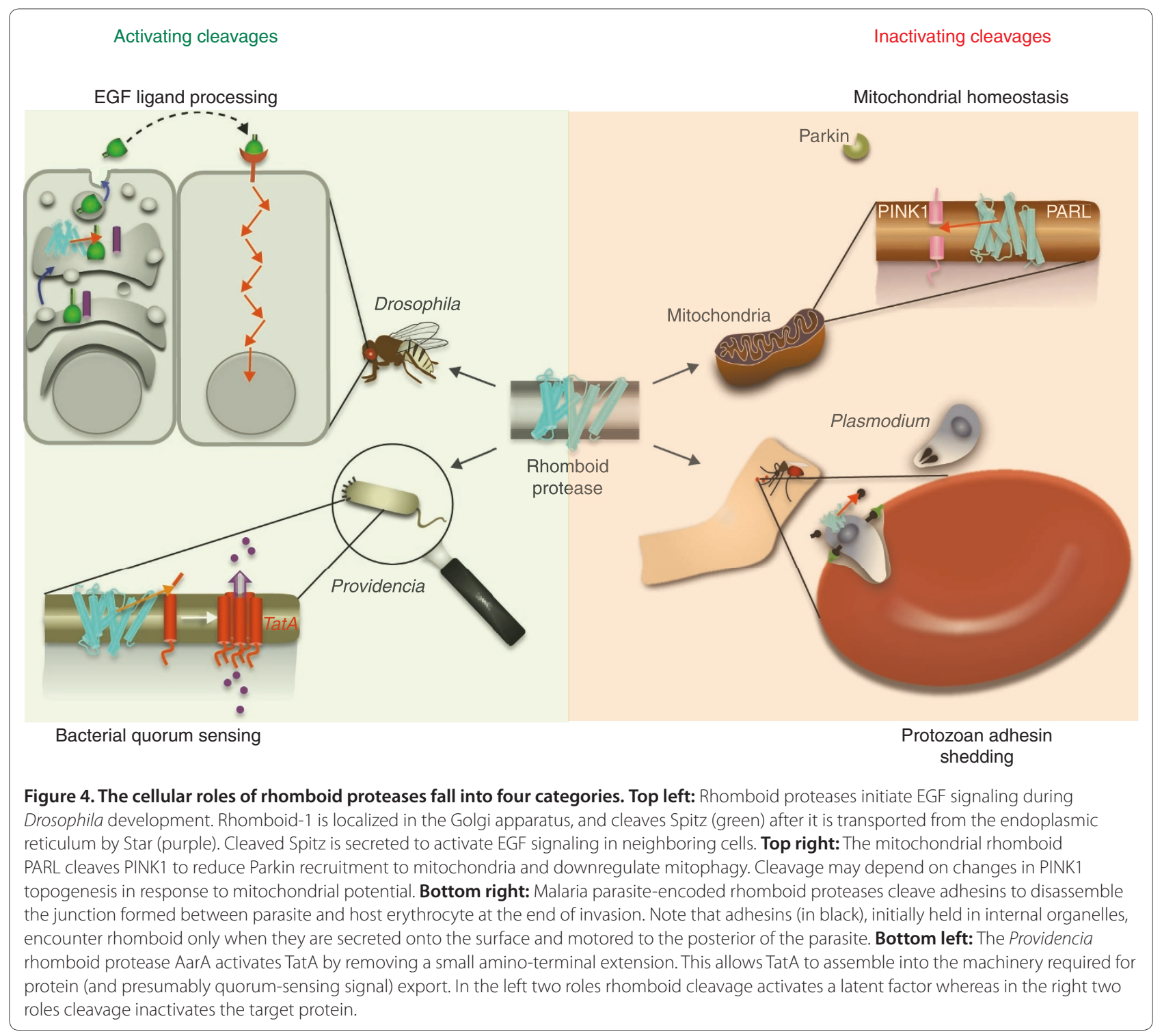

\section{Mechanism}

Perhaps the most powerful, yet subtle, guiding principle that can be deduced from the near ubiquity of rhomboid proteases is that they possess a biochemical property that is both very rare and highly useful: but what? Solving this riddle requires understanding the enzymatic features of rhomboid proteases, and remarkable progress has been made towards these goals (reviewed in [38]).

There is now proof beyond doubt that rhomboid enzymes are serine proteases. This includes reconstitution of proteolysis with pure proteins [17,19], protease inhibitor profiling $[16,17,76]$, extensive analysis of residues essential for activity $[16,18,19,77]$, and structural visualization of catalytic residues and with a covalently bound inhibitor [34-37,78]. Moreover, the initial paradox of how water is delivered to the membrane-immersed active site for hydrolysis was largely addressed by structural analyses [34-37]: the active site lies submerged about 10 A below the presumed membrane surface, but with an open cavity above the active site for water access (Figure 3).

Structure-function analyses of rhomboid proteases have also revealed several unusual proteolytic properties that make them unlike most serine proteases. These differences are clear evidence of convergent evolution to a serine protease mechanism down an independent path. First, structural analysis indicates that nucleophilic catalysis is achieved by a histidine-serine catalytic pair, rather than the more common aspartate-histidine-serine catalytic triad [34-37]. Catalytic dyads have been noted in 
a minority of exceptional serine proteases [79]. The identity of the residues that stabilize the oxyanion transition state is uncertain, but this stabilization is most likely mediated by asparagine and/or histidine sidechains [36,78] (Figure 3). Use of an asparagine for oxyanion stabilization is uncommon but strikingly analogous to the mechanism of the conventional serine protease subtilisin [80].

The third unusual catalytic property of rhomboid proteases relates to the direction in which substrates lie across the active site cleft relative to the catalytic residues. Although initially thought to be similar to nearly all other serine proteases [34,37], identification of the substrate gate on the opposite side of GlpG relative to expectation mandated that substrates approach the catalytic residues from the so-called 'si' face [35,77,81]. This stereochemical arrangement is very uncommon and had only been encountered in $\alpha / \beta$-hydrolyses [82]. Consistent with this stereochemistry are rhomboid's resistance to most canonical serine protease inhibitors and a weak but specific sensitivity to monocyclic $\beta$-lactams $[16,17,76]$. It should be stressed that the definitive evidence for substrate orientation, identity of the oxyanion hole, and the nature of substrate stabilization await a co-structure with a peptide substrate.

Rhomboid proteases have been studied largely within the framework of an established serine protease precedent as a way to interpret rhomboid mechanism, which is instructive but does not help to understand how they are different. Although deciphering the specifics of the catalytic chemistry is essential for designing effective inhibitors, the key functional properties of rhomboid enzymes that are relevant to the cell are unlikely to be determined by its catalytic mechanism. These defining features most likely result from membrane-immersion of the enzyme, and more recent investigations have started to study rhomboid proteases as integral membrane proteins directly.

The greatest impact of membrane immersion is on how substrates and rhomboid proteases behave (as reviewed in [38]). The closed ring of TM segments observed in the first crystal structure suggested that something must move to clear a path for lateral substrate entry [34-37]. Only mutations that weaken TM5 packing with TM2 were found to enhance protease activity by up to ten-fold, thereby identifying the gate functionally $[77,81,83]$. This dramatic enhancement also revealed that gate opening is the rate-limiting step for intramembrane proteolysis. Molecular dynamics simulations and structural analysis in a bicelle also suggest membrane thinning surrounding GlpG, but its mechanistic implications remain unclear $[84,85]$. Investigating the role of the membrane in greater detail promises to reveal the defining features of the rhomboid proteolysis system.

\section{Frontiers}

The rhomboid gene was identified in the Drosophila screens of the late 1970s and early 1980s [8], and it was cloned and sequenced about a decade later [10]. It took another decade, until 2001, for its biochemical function as an intramembrane serine protease to be revealed [16]. It has now been a decade since that turning point, and advances in the intervening period have culminated in rhomboid proteases becoming widely regarded as the best understood of all intramembrane proteases [38]. Biochemical insights and defined roles in parasitic protozoa (reviewed in [6]) place rhomboid study on the cusp of becoming applicable in a therapeutic setting. A major lingering obstacle is a rudimentary understanding of its unusual enzymatic mechanism, but these questions are being pursued intensively, and momentum towards a sophisticated understanding is building [38].

By contrast, defining the cellular roles of rhomboid proteases has been a slow process [86]. Although even early biochemical insights have led to the identification of substrates that can be cleaved, whether these candidates are indeed physiological targets, and if so, whether they truly represent a major rhomboid function, remain unknown. For example, although the study of human RHBDL2 over the past 7 years has uncovered at least three well-cleaved substrates (thrombomodulin [24], B-type ephrins [87], and EGF [48]), it is still unclear which, if any, are actual physiological targets, and whether cleavage represents a bona fide contribution to cellular function. Perhaps the most humbling example is $E$. coli GlpG, whose atomic details have been revealed in over a dozen structures and countless mutants, yet its cellular function remains a complete mystery [88]. In reality, it is not the ability to find substrate candidates but rather their validation that has proven to be the bottleneck in these studies. Refining search algorithms is unlikely to contribute much towards solving this problem. The urgent need is for approaches with which to study enzymes under physiological settings on a higher throughput scale. This, in turn, will focus biochemical investigations by providing physiological targets and new functional contexts.

\section{Competing interests}

The authors declare that they have no competing interests.

\section{Acknowledgements}

We are grateful to Rosanna Baker for help with illustrating Figure 4. Work in our laboratory is supported by the Howard Hughes Medical Institute, and the David and Lucile Packard Foundation.

Published: 27 October 2011

\section{References}

1. Sakai J, Duncan EA, Rawson RB, Hua X, Brown MS, Goldstein JL: Sterolregulated release of SREBP-2 from cell membranes requires two sequential cleavages, one within a transmembrane segment. Cell 1996, 85:1037-1046. 
2. Rawson RB, Zelenski NG, Nijhawan D, Ye J, Sakai J, Hasan MT, Chang TY, Brown MS, Goldstein JL: Complementation cloning of S2P, a gene encoding a putative metalloprotease required for intramembrane cleavage of SREBPs. Mol Cell 1997, 1:47-57.

3. Wolfe MS, Xia W, Ostaszewski BL, Diehl TS, Kimberly WT, Selkoe DJ: Two transmembrane aspartates in presenilin-1 required for presenilin endoproteolysis and gamma-secretase activity. Nature 1999, 398:513-517.

4. Selkoe DJ, Wolfe MS: Presenilin: running with scissors in the membrane. Cell 2007, 131:215-221.

5. Haffner C, Haass C: Cellular functions of gamma-secretase-related proteins. Neurodegener Dis 2006, 3:284-289.

6. Urban S: Making the cut: central roles of intramembrane proteolysis in pathogenic microorganisms. Nat Rev Microbiol 2009, 7:411-423.

7. Osborne TF, Espenshade PJ: Evolutionary conservation and adaptation in the mechanism that regulates SREBP action: what a long, strange tRIP it's been. Genes Dev 2009, 23:2578-2591.

8. Jurgens G, Wieschaus E, Nusslein-Volhard C, Kluding H: Mutations affecting the pattern of the larval cuticle in Drosophila melanogaster. Roux Arch Dev Biol 1984, 193:267-282

9. Mayer U, Nusslein-Volhard C: A group of genes required for pattern formation in the ventral ectoderm of the Drosophila embryo. Genes Dev 1988, 2:1496-1511.

10. Bier $E$, Jan $L Y$, Jan $Y N$ : rhomboid, a gene required for dorsoventral axis establishment and peripheral nervous system development in Drosophila melanogaster. Genes Dev 1990, 4:190-203.

11. Rutledge BJ, Zhang K, Bier E, Jan YN, Perrimon N: The Drosophila spitz gene encodes a putative EGF-like growth factor involved in dorsal-ventral axis formation and neurogenesis. Genes Dev 1992, 6:1503-1517.

12. Wasserman JD, Urban S, Freeman M: A family of rhomboid-like genes: Drosophila rhomboid-1 and roughoid/rhomboid-3 cooperate to activate EGF receptor signalling. Genes Dev 2000, 14:1651-1663.

13. Guichard A, Biehs B, Sturtevant MA, Wickline L, Chacko J, Howard K, Bier E: rhomboid and Star interact synergistically to promote EGFR/MAPK signaling during Drosophila wing vein development. Development 1999, 126:2663-2676

14. Wasserman JD, Freeman M: An autoregulatory cascade of EGF receptor signalling patterns the Drosophila egg. Cell 1998, 95:355-364.

15. Schweitzer R, Shaharabany M, Seger R, Shilo B-Z: Secreted Spitz triggers the DER signalling pathway and is a limiting component in embryonic ventral ectoderm determination. Genes Dev 1995, 9:1518-1529.

16. Urban S, Lee JR, Freeman M: Drosophila rhomboid-1 defines a family of putative intramembrane serine proteases. Cell 2001, 107:173-182

17. Urban S, Wolfe MS: Reconstitution of intramembrane proteolysis in vitro reveals that pure rhomboid is sufficient for catalysis and specificity. Proc Natl Acad Sci U S A 2005, 102:1883-1888.

18. Lemberg MK, Menendez J, Misik A, Garcia M, Koth CM, Freeman M: Mechanism of intramembrane proteolysis investigated with purified rhomboid proteases. EMBO J 2005, 24:464-472.

19. Maegawa S, Ito K, Akiyama Y: Proteolytic action of GlpG, a rhomboid protease in the Escherichia coli cytoplasmic membrane. Biochemistry (Mosc) 2005, 44:13543-13552.

20. Urban S, Schlieper D, Freeman M: Conservation of intramembrane proteolytic activity and substrate specificity in eukaryotic and prokaryotic Rhomboids. Curr Biol 2002, 12:1507-1512.

21. Koonin EV, Makarova KS, Rogozin IB, Davidovic L, Letellier MC, Pellegrini L: The rhomboids: a nearly ubiquitous family of intramembrane serine proteases that probably evolved by multiple ancient horizontal gene transfers. Genome Bio/ 2003, 4:R19.

22. Lemberg MK, Freeman M: Functional and evolutionary implications of enhanced genomic analysis of rhomboid intramembrane proteases. Genome Res 2007, 17:1634-1646

23. Kanaoka MM, Urban S, Freeman M, Okada K: An Arabidopsis Rhomboid homolog is an intramembrane protease in plants. FEBS Lett 2005, 579:5723-5728.

24. Lohi O, Urban S, Freeman M: Diverse substrate recognition mechanisms for rhomboids; thrombomodulin is cleaved by mammalian rhomboids. Curr Biol 2004, 14:236-241.

25. Brossier F, Jewett TJ, Sibley LD, Urban S: A spatially localized rhomboid protease cleaves cell surface adhesins essential for invasion by Toxoplasma. Proc Natl Acad Sci U S A 2005, 102:4146-4151.

26. Baker RP, Wijetilaka R, Urban S: Two Plasmodium Rhomboid proteases preferentially cleave different adhesins implicated in all invasive stages of malaria. PLoS Pathog 2006, 2:e113.

27. Oberai A, Joh NH, Pettit FK, Bowie JU: Structural imperatives impose diverse evolutionary constraints on helical membrane proteins. Proc Natl Acad Sci USA 2009, 106:17747-17750.

28. McQuibban GA, Saurya S, Freeman M: Mitochondrial membrane remodelling regulated by a conserved rhomboid protease. Nature 2003, 423:537-541.

29. Cipolat S, Rudka T, Hartmann D, Costa V, Serneels L, Craessaerts K, Metzger K, Frezza C, Annaert W, D'Adamio L, Derks C, Dejaegere T, Pellegrini L, D'Hooge R, Scorrano L, De Strooper B: Mitochondrial rhomboid PARL regulates cytochrome c release during apoptosis via OPA1-dependent cristae remodeling. Cell 2006, 126:163-175.

30. McQuibban GA, Lee JR, Zheng L, Juusola M, Freeman M: Normal mitochondrial dynamics requires rhomboid-7 and affects Drosophila lifespan and neuronal function. Curr Biol 2006, 16:982-989.

31. Karakasis $K$, Taylor D, Ko K: Uncovering a link between a plastid translocon component and rhomboid proteases using yeast mitochondria-based assays. Plant Cell Physiol 2007, 48:655-661.

32. Strisovsky K, Sharpe HJ, Freeman M: Sequence-specific intramembrane proteolysis: identification of a recognition motif in rhomboid substrates. Mol Cell 2009, 36:1048-1059.

33. Urban S, Freeman M: Substrate specificity of rhomboid intramembrane proteases is governed by helix-breaking residues in the substrate transmembrane domain. Mol Cell 2003, 11:1425-1434.

34. Wang $\mathrm{Y}$, Zhang $\mathrm{Y}, \mathrm{Ha}$ Y: Crystal structure of a rhomboid family intramembrane protease. Nature 2006, 444:179-180.

35. Wu Z, Yan N, Feng L, Oberstein A, Yan H, Baker RP, Gu L, Jeffrey PD, Urban S, Shi Y: Structural analysis of a rhomboid family intramembrane protease reveals a gating mechanism for substrate entry. Nat Struct Mol Biol 2006, 13:1084-1091.

36. Ben-Shem A, Fass D, Bibi E: Structural basis for intramembrane proteolysis by rhomboid serine proteases. Proc Natl Acad Sci U S A 2007, 104:462-466.

37. Lemieux MJ, Fischer SJ, Cherney MM, Bateman KS, James MN: The crystal structure of the rhomboid peptidase from Haemophilus influenzae provides insight into intramembrane proteolysis. Proc Natl Acad Sci U S A 2007, 104:750-754

38. Urban S: Taking the plunge: integrating structural, enzymatic and computational insights into a unified model for membrane-immersed rhomboid proteolysis. Biochem J 2010, 425:501-512.

39. Jeyaraju DV, McBride HM, Hill RB, Pellegrini L: Structural and mechanistic basis of Parl activity and regulation. Cell Death Differ 2011, 18:1531-1539.

40. Sheiner L, Dowse TJ, Soldati-Favre D: Identification of trafficking determinants for polytopic rhomboid proteases in Toxoplasma gondii. Traffic 2008, 9:665-677.

41. Zettl M, Adrain C, Strisovsky K, Lastun V, Freeman M: Rhomboid family pseudoproteases use the ER quality control machinery to regulate intercellular signaling. Cell 2011, 145:79-91.

42. Lilley BN, Ploegh HL: A membrane protein required for dislocation of misfolded proteins from the ER. Nature 2004, 429:834-840.

43. Ye Y, Shibata Y, Yun C, Ron D, Rapoport TA: A membrane protein complex mediates retro-translocation from the ER lumen into the cytosol. Nature 2004, 429:841-847.

44. Sato BK, Hampton RY: Yeast Derlin Dfm 1 interacts with Cdc48 and functions in ER homeostasis. Yeast 2006, 23:1053-1064

45. Knop M, Finger A, Braun T, Hellmuth K, Wolf DH: Der1, a novel protein specifically required for endoplasmic reticulum degradation in yeast. EMBO J 1996, 15:753-763.

46. Lee JR, Urban S, Garvey CF, Freeman M: Regulated intracellular ligand transport and proteolysis control EGF signal activation in Drosophila. Cell 2001, 107:161-171

47. Dutt A, Canevascini S, Froehli-Hoier E, Hajnal A: EGF signal propagation during C. elegans vulval development mediated by ROM-1 rhomboid. PLoS Biol 2004, 2:e334.

48. Adrain C, Strisovsky K, Zettl M, Hu L, Lemberg MK, Freeman M: Mammalian EGF receptor activation by the rhomboid protease RHBDL2. EMBO Rep 2011, 12:421-427.

49. Wang Y, Guan X, Fok KL, Li S, Zhang X, Miao S, Zong S, Koide SS, Chan HC, Wang L: A novel member of the Rhomboid family, RHBDD1, regulates BIK-mediated apoptosis. Cell Mol Life Sci 2008, 65:3822-3829.

50. Zou H, Thomas SM, Yan ZW, Grandis JR, Vogt A, Li LY: Human rhomboid 
family-1 gene RHBDF1 participates in GPCR-mediated transactivation of EGFR growth signals in head and neck squamous cancer cells. FASEB J 2009, 23:425-432

51. Yan Z, Zou H, Tian F, Grandis JR, Mixson AJ, Lu PY, Li LY: Human rhomboid family-1 gene silencing causes apoptosis or autophagy to epithelial cancer cells and inhibits xenograft tumor growth. Mol Cancer Ther 2008, 7:1355-1364.

52. Herlan M, Vogel F, Bornhovd C, Neupert W, Reichert AS: Processing of Mgm by the rhomboid-type protease Pcp1 is required for maintenance of mitochondrial morphology and of mitochondrial DNA. J Biol Chem 2003, 278:27781-27788

53. Sesaki H, Southard SM, Hobbs AE, Jensen RE: Cells lacking Pcp1p/Ugo2p, a rhomboid-like protease required for Mgm $1 p$ processing, lose mtDNA and mitochondrial structure in a Dnm1p-dependent manner, but remain competent for mitochondrial fusion. Biochem Biophys Res Commun 2003, 308:276-283.

54. Herlan M, Bornhovd C, Hell K, Neupert W, Reichert AS: Alternative topogenesis of Mgm1 and mitochondrial morphology depend on ATP and a functional import motor. J Cell Biol 2004, 165:167-173.

55. Whitworth AJ, Lee JR, Ho VM, Flick R, Chowdhury R, McQuibban GA: Rhomboid-7 and HtrA2/Omi act in a common pathway with the Parkinson's disease factors Pink1 and Parkin. Dis Model Mech 2008, 1:168-174; discussion 173.

56. Jin SM, Lazarou M, Wang C, Kane LA, Narendra DP, Youle RJ: Mitochondrial membrane potential regulates PINK1 import and proteolytic destabilization by PARL. J Cell Biol 2010, 191:933-942.

57. Deas E, Plun-Favreau H, Gandhi S, Desmond H, Kjaer S, Loh SH, Renton AE, Harvey RJ, Whitworth AJ, Martins LM, Abramov AY, Wood NW: PINK1 cleavage at position $\mathrm{A} 103$ by the mitochondrial protease PARL. Hum Mol Genet 2010, 20:867-879.

58. Shi G, Lee JR, Grimes DA, Racacho L, Ye D, Yang H, Ross OA, Farrer M McQuibban GA, Bulman DE: Functional alteration of PARL contributes to mitochondrial dysregulation in Parkinson's disease. Hum Mol Genet 2011, 20:1966-1974

59. Chao JR, Parganas E, Boyd K, Hong CY, Opferman JT, Ihle JN: Hax1-mediated processing of $\mathrm{HtrA} 2$ by Parl allows survival of lymphocytes and neurons. Nature 2008, 452:98-102.

60. Civitarese AE, MacLean PS, Carling S, Kerr-Bayles L, McMillan RP, Pierce A, Becker TC, Moro C, Finlayson J, Lefort N, Newgard CB, Mandarino L, Cefalu W, Walder K, Collier GR, Hulver MW, Smith SR, Ravussin E: Regulation of skeletal muscle oxidative capacity and insulin signaling by the mitochondrial rhomboid protease PARL. Cell Metab 2010, 11:412-426.

61. Rather PN, Orosz E: Characterization of aarA, a pleiotrophic negative regulator of the 2'-N-acetyltransferase in Providencia stuartii. J Bacteriol 1994, 176:5140-5144.

62. Rather PN, Ding X, Baca-DeLancey RR, Siddiqui S: Providencia stuartii genes activated by cell-to-cell signaling and identification of a gene required for production or activity of an extracellular factor. J Bacterio/ 1999, 181:7185-7191.

63. Gallio M, Kylsten P: Providencia may help find a function for a novel, widespread protein family. Curr Biol 2000, 10:R693-694.

64. Gallio M, Sturgill G, Rather P, Kylsten P: A conserved mechanism for extracellular signaling in eukaryotes and prokaryotes. Proc Natl Acad SCi US A 2002, 99:12208-12213.

65. Urban S: Rhomboid proteins: conserved membrane proteases with divergent biological functions. Genes Dev 2006, 20:3054-3068

66. Stevenson LG, Strisovsky K, Clemmer KM, Bhatt S, Freeman M, Rather PN: Rhomboid protease AarA mediates quorum-sensing in Providencia stuartii by activating TatA of the twin-arginine translocase. Proc Natl Acad Sci U S A 2007, 104:1003-1008.

67. Sibley LD: Invasion and intracellular survival by protozoan parasites. Immunol Rev 2011, 240:72-91.

68. Dowse TJ, Pascall JC, Brown KD, Soldati D: Apicomplexan rhomboids have a potential role in microneme protein cleavage during host cell invasion. Int J Parasitol 2005, 35:747-756.

69. O'Donnell RA, Hackett F, Howell SA, Treeck M, Struck N, Krnajski Z, WithersMartinez C, Gilberger TW, Blackman MJ: Intramembrane proteolysis mediates shedding of a key adhesin during erythrocyte invasion by the malaria parasite. J Cell Biol 2006, 174:1023-1033.
70. Srinivasan P, Coppens I, Jacobs-Lorena M: Distinct roles of Plasmodium rhomboid 1 in parasite development and malaria pathogenesis. PLOS Pathog 2009, 5:e1000262.

71. Buguliskis JS, Brossier F, Shuman J, Sibley LD: Rhomboid 4 (ROM4) affects the processing of surface adhesins and facilitates host cell invasion by Toxoplasma gondii. PLoS Pathog 2010, 6:e1000858.

72. Santos JM, Ferguson DJ, Blackman MJ, Soldati-Favre D: Intramembrane cleavage of AMA1 triggers Toxoplasma to switch from an invasive to a replicative mode. Science 2011, 331:473-477.

73. Baxt LA, Baker RP, Singh U, Urban S: An Entamoeba histolytica rhomboid protease with atypical specificity cleaves a surface lectin involved in phagocytosis and immune evasion. Genes Dev 2008, 22:1636-1646

74. Baxt LA, Rastew E, Bracha R, Mirelman D, Singh U: Downregulation of an Entamoeba histolytica rhomboid protease reveals roles in regulating parasite adhesion and phagocytosis. Euk Cell 2010, 9:1283-1293.

75. Brossier F, Starnes GL, Beatty WL, Sibley LD: Microneme rhomboid protease TgROM1 is required for efficient intracellular growth of Toxoplasma gondii. Eukaryotic cell 2008, 7:664-674.

76. Pierrat OA, Strisovsky K, Christova Y, Large J, Ansell K, Bouloc N, Smiljanic E, Freeman M: Monocyclic beta-lactams are selective, mechanism-based inhibitors of rhomboid intramembrane proteases. ACS Chem Biol 2011, 6:325-335.

77. Baker RP, Young K, Feng L, Shi Y, Urban S: Enzymatic analysis of a rhomboid intramembrane protease implicates transmembrane helix 5 as the lateral substrate gate. Proc Natl Acad Sci U S A 2007, 104:8257-8262.

78. Vinothkumar KR, Strisovsky K, Andreeva A, Christova Y, Verhelst S, Freeman M: The structural basis for catalysis and substrate specificity of a rhomboid protease. EMBO J 2010, 29:3797-3809.

79. Ekici OD, Paetzel M, Dalbey RE: Unconventional serine proteases: variations on the catalytic Ser/His/Asp triad configuration. Protein Sci 2008, 17:2023-2037.

80. Polgar L: The catalytic triad of serine peptidases. Cell Mol Life Sci 2005, 62:2161-2172.

81. Brooks CL, Lazareno-Saez C, Lamoureux JS, Mak MW, Lemieux MJ: Insights into substrate gating in $\mathrm{H}$. influenzae rhomboid. J Mol Biol 2011, 407:687-697.

82. Paetzel M, Dalbey RE, Strynadka NC: Crystal structure of a bacterial signal peptidase in complex with a beta-lactam inhibitor. Nature 1998, 396:186-190.

83. Urban S, Baker RP: In vivo analysis reveals substrate-gating mutants of a rhomboid intramembrane protease display increased activity in living cells. Biol Chem 2008, 389:1107-1115.

84. Bondar AN, del Val C, White SH: Rhomboid protease dynamics and lipid interactions. Structure 2009, 17:395-405.

85. Vinothkumar KR: Structure of rhomboid protease in a lipid environment J Mol Biol 2011, 407:232-247.

86. Freeman M: Rhomboids: 7 years of a new protease family. Semin Cell Dev Biol 2009, 20:231-239.

87. Pascall JC, Brown KD: Intramembrane cleavage of ephrinB3 by the human rhomboid family protease, RHBDL2. Biochem Biophys Res Commun 2004, 317:244-252

88. Clemmer KM, Sturgill GM, Veenstra A, Rather PN: Functional characterization of Escherichia coli GlpG and additional rhomboid proteins using an aarA mutant of Providencia stuartii. J Bacterio/ 2006, 188:3415-3419.

89. Urban S, Lee JR, Freeman M: A family of Rhomboid intramembrane proteases activates all membrane-tether EGF ligands in Drosophila. EMBO J 2002, 21:4277-4286.

90. Schulz C, Wood CG, Jones DL, Tazuke SI, Fuller MT: Signaling from germ cells mediated by the rhomboid homolog stet organizes encapsulation by somatic support cells. Development 2002, 129:4523-4534.

91. Urban S, Brown G, Freeman M: EGF receptor signaling protects smoothcuticle cells from apoptosis during Drosophila ventral epidermis development. Development 2004, 131:1835-1845.

\section{doi:10.1186/gb-2011-12-10-231}

Cite this article as: Urban S, Dickey SW: The rhomboid protease family: a decade of progress on function and mechanism. Genome Biology 2011, 12:231. 